\title{
Dynamical properties of Bianchi-I spacetimes in $f(R)$ gravity
}

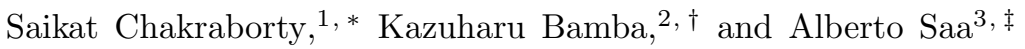 \\ ${ }^{1}$ Department of Physics, Indian Institute of Technology, Kanpur 208016, India \\ ${ }^{2}$ Division of Human Support System, Faculty of Symbiotic Systems Science, \\ Fukushima University, Fukushima 960-1296, Japan \\ ${ }^{3}$ Department of Applied Mathematics, University of Campinas, 13083-859 Campinas, SP, Brazil.
}

(Dated: March 13, 2019)

\begin{abstract}
We present a dynamical analysis in terms of new expansion-normalized variables for homogeneous and anisotropic Bianchi-I spacetimes in $f(R)$ gravity in the presence of anisotropic matter. With a suitable choice of the evolution parameter, the Einstein's equations are reduced to an autonomous 5dimensional system of ordinary differential equations for the new variables. Further restrictions lead to considerable simplifications. For instance, we show that for a large class of functions $f(R)$, which includes several cases commonly considered in the literature, all the fixed points are polynomial roots, and hence they can be determined with good accuracy and classified for stability. Moreover, typically for these cases, any fixed point corresponding to isotropic solutions in the presence of anisotropic matter will be unstable. The assumption of a perfect fluid as source and or the vacuum cases imply some dimensional reductions and even more simplifications. In particular, we find that the vacuum solutions of $f(R)=R^{1+\delta}$, with $\delta$ a constant, are governed by an effective bi-dimensional phase space which can be analytically constructed, leading to an exactly soluble dynamics. Finally, we demonstrate that several results already reported in the literature can be re-obtained in a more direct and easy way by exploring our dynamical formulation.
\end{abstract}

\section{INTRODUCTION}

The observable universe can be described by the homogeneous and isotropic Friedmann-Lemaitre-RobertsonWalker (FLRW) metric with a high degree of accuracy. Any inhomogeneity and anisotropy observed is of very small scale and can be satisfactorily described by cosmological perturbation theory, see, for instance, [1, 2]. Cosmological perturbations are believed to be generated due to quantum fluctuations in the very early universe. On the other hand, the observation of the large scale isotropy of the present universe suggests that the dynamics of the early universe must include an inherent isotropization mechanism. Since the dynamics of the universe in any relativistic theory of gravity is essentially a non-linear system of ordinary differential equations, they can be described, in general, by constructing a set of autonomous first order system together with an energy constraint, see 3, 4, for instance, for comprehensive reviews on the subject. We can therefore state, in the language of nonlinear dynamics, that the isotropic solution describing our universe must arise as an attractor in the space of more general anisotropic solutions at some early epoch of the universe. The most successful paradigm about the early universe, namely the inflationary scenarios [5, 6], assumes the existence of a brief epoch of rapid, almost exponential expansion at the very early stage of the universe. An eternally inflating universe is usually described with a de Sitter solution, characterized by a constant value of the Hubble parameter $H$. For cosmo-

\footnotetext{
* snilch@iitk.ac.in

$\dagger$ bamba@sss.fukushima-u.ac.jp

$\ddagger$ asaa@ime.unicamp.br
}

logically realistic inflationary scenarios, however, a quasi de Sitter epoch is used, wherein the Hubble parameter gradually decreases, leading to a smooth end to the inflation. A very successful model for an inflationary period was given by Starobinsky [7, which employed an $R+\alpha R^{2}$ Lagrangian for gravity with a positive value of $\alpha$. Indeed, quantum corrections to General Relativity (GR) leading to modifications in Einstein-Hilbert Lagrangian of this type may not be something unexpected in the high curvature regime as in the early universe. The Starobinsky's inflationary scenario, which is now receiving considerable attention due to its compatibility with the Planck 2018 Results [8, 9], is based on the existence of pure vacuum de-Sitter solution in $R^{2}$ gravity, whereas the linear term in $R$ actually plays the role of making the evolution a quasi de Sitter one and supressing the inflation after about 70 e-foldings, see [10, 11] for further references.

Here, we will be concerned with the standard $f(R)$ modified theory of gravity, which is governed by the action

$$
S_{=} \frac{1}{2 \kappa} \int d^{4} x \sqrt{-g} f(R)+S_{M}
$$

where $\kappa=8 \pi G, c=\hbar=1$, and $S_{M}$ stands for the usual matter contributions to the total action. The $f(R)$ gravity has been intensively studied as an alternative description to dark energy and the late acceleration of the universe, see [12 14] for instance. For recent comprehensive reviews, see [15 21]. Most of the works on the dynamics of (1) typically assume a homogeneous and isotropic FLRW model to start with, and do not take metric anisotropy into account, see, for instance, [22 29]. Nevertheless, the dynamics of metric shear for the case of $R^{n}$ gravity in vacuum and in presence of an isotropic fluid have previously been studied using the $(1+3)$ covari- 
ant formalism [30, 31], but the case involving anistropic matter was still lacking in the literature.

In this work, we have extended the so-called expansionnormalized variables [3] to write down the dynamical equations of (1), for a homogeneous and anisotropic Bianchi-I metric in the presence of an anisotropic fluid, as a 5-dimensional system of ordinary differential equations. We will show that some further assumptions may lead to considerable simplifications in the equations, and for several examples we end up with analytically soluble systems. For the sake of illustration, we consider explicitly the case of $f(R)=R^{1+\delta}$. First, we show that the formulation of [30, 31] is recovered in the isotropic matter limit. Then, we rederive, in a simpler and more direct way, several known results as the existence of vacuum Kasner-like solutions for $-\frac{1}{2} \leq \delta \leq \frac{1}{4}$ [32, 33] and some uniqueness and stability properties of the Starobinsky's isotropic inflationary scenario in $R^{2}$ gravity [34 36]. We also obtain several explicit new results, as the complete dynamical characterization of vacuum solutions for the case $f(R)=R^{1+\delta}$, and the instability of isotropic solutions in the presence of anisotropic matter for all $f(R)$ with polynomials fixed points.

The paper is organized as follows. In section II, we present the dynamical equations for a Bianchi-I cosmology for (1) in the presence of an anisotropic fluid. The isotropic fluid limit is discussed, and we also introduce the new expansion-normalized variables for the system. Section III is devoted for the applications of our dynamical approach in several explicit examples, and the last section is left for some concluding remarks.

\section{BIANCHI-I COSMOLOGY IN $f(R)$ GRAVITY WITH ANISOTROPIC FLUID}

We will consider the homogeneous and anisotropic Bianchi-I metric, which can be conveniently cast for our purposes in the following form 37,39

$$
d s^{2}=-d t^{2}+a^{2}(t) \sum_{i=1}^{3} e^{2 \beta_{i}(t)}\left(d x^{i}\right)^{2}
$$

where $a(t)$ is the average scale factor and the three functions $\beta_{i}$, which characterize the anisotropies, are such that $\beta_{1}+\beta_{2}+\beta_{3}=0$. In our studies, it will be more convenient to employ the variables

$$
\beta_{ \pm}=\beta_{1} \pm \beta_{2}
$$

The total amount of anisotropy in the metric (2) is given by the quantity

$$
\sigma^{2}=\dot{\beta}_{1}^{2}+\dot{\beta}_{2}^{2}+\dot{\beta}_{3}^{2}=\frac{3}{2} \dot{\beta}_{+}^{2}+\frac{1}{2} \dot{\beta}_{-}^{2} .
$$

For $\sigma=0$, one can show that the spatial coordinates $x^{i}$ can be suitably rescaled to recast the Bianchi-I metric in the standard FLRW form. The Ricci scalar for the metric (2) reads

$$
R=6 \dot{H}+12 H^{2}+\sigma^{2}
$$

where the average Hubble parameter $H$ is given by the standard expression

$$
H=\frac{\dot{a}}{a} .
$$

We will assume also the presence of an anisotropic barotropic fluid with energy momentum tensor parametrized as 40

$$
T_{\mu}^{\nu}=\operatorname{diag}\left(-\rho, p_{1}, p_{2}, p_{3}\right)=\operatorname{diag}\left(-\rho, \omega_{1} \rho, \omega_{2} \rho, \omega_{3} \rho\right),
$$

and we define the anisotropic equation of state as

$$
p_{i}=\left(\omega+\mu_{i}\right) \rho,
$$

with $i=1,2,3$, where $\omega$ is the average barotropic parameter and $\omega_{i}=\omega+\mu_{i}$, with $\mu_{1}+\mu_{2}+\mu_{3}=0$ by construction. As in [39], we will parameterize our fluid by the constants $\omega$ and $\mu_{ \pm}=\mu_{1} \pm \mu_{2}$.

The dynamics of the Bianchi-I metric (2) under $f(R)$ gravity action (1), in the presence of and anisotropic barotropic fluid with energy-momentum tensor (7), can be described by the following set of equations 39],

$$
\begin{gathered}
3 H^{2}=\frac{\kappa}{f^{\prime}}\left(\rho+\frac{R f^{\prime}-f}{2 \kappa}-\frac{3 H f^{\prime \prime} \dot{R}}{\kappa}\right)+\frac{\sigma^{2}}{2} \\
2 \dot{H}+3 H^{2}=-\frac{\kappa}{f^{\prime}}\left(\omega \rho+\frac{\dot{R}^{2} f^{\prime \prime \prime}+(2 H \dot{R}+\ddot{R}) f^{\prime \prime}}{\kappa}\right. \\
\left.-\frac{R f^{\prime}-f}{2 \kappa}\right)-\frac{\sigma^{2}}{2} \\
\ddot{\beta_{ \pm}}+\left(3 H+\frac{\dot{R} f^{\prime \prime}}{f^{\prime}}\right) \dot{\beta}_{ \pm}=\frac{\kappa \rho}{F} \mu_{ \pm} \\
\dot{\rho}+(3 H(1+\omega)+\boldsymbol{\delta} \cdot \dot{\boldsymbol{\beta}}) \rho=0
\end{gathered}
$$

where $i=1,2,3$, and

$$
\boldsymbol{\delta} \cdot \dot{\boldsymbol{\beta}}=\mu_{1} \dot{\beta}_{1}+\mu_{2} \dot{\beta}_{2}+\mu_{3} \dot{\beta}_{3}=\frac{3}{2} \mu_{+} \dot{\beta}_{+}+\frac{1}{2} \mu_{-} \dot{\beta}_{-} .
$$

Notice that in the presence of a perfect fluid, we will have $\mu_{+}=\mu_{-}=0$ and the two equations 11 for $\beta_{+}$and $\beta_{-}$ can be substituted with

$$
\dot{\sigma}+\left(3 H+\frac{\dot{R} f^{\prime \prime}}{f^{\prime}}\right) \sigma=0 .
$$

In this case, there is no anisotropy in the matter sector and the single variable $\sigma$ is sufficient to describe the total amount of metric anisotropy in the system. As we can see, in general, we will have four functions of time 
$H(t), \rho(t), \beta_{ \pm}(t)$ governing the dynamics. The existence of the constraint equation (9) implies that only three of them are indeed independent. Without loss of generality, we can choose them to be, for instance, $H(t)$ and $\beta_{ \pm}(t)$. Given some specific form of the function $f(R)$, they can be determined by solving equations $(10)$ and (11). The fluid energy density $\rho(t)$ can then be found using the energy constraint $(9)$.

\section{A. The expansion-normalized variables}

The traditional expansion-normalized variables were initially introduced for a better dynamical analysis of the standard FLRW model, see [3] for instance. Here, we will expand the variables already introduced in in 30, 31] to include the case of the anisotropic barotropic fluid (7). In this regard, let us introduce the monotonically increasing variable

$$
N=\epsilon \ln a
$$

known as the logarithmic time, where $\epsilon$ is defined to be +1 for expanding universe and -1 for a contracting one. Without loss of generality, we choose the scale factor at $t=0$ to be $a_{0}=1$. Therefore, as time progresses in the forward (positive) direction, the logarithmic time $N$ becomes positive and goes towards $+\infty$ in case of both expanding and contracting universes. One can notice that

$$
\dot{N}=\epsilon H,
$$

so that $\dot{N}$ is effectively always positive, justifying the use of $N$ as the dimensionless evolution variable for both expanding and contracting universes. On the other hand, around a bounce or a turnaround point, this argument is not valid though and the expanding and contracting branches must be considered separately.

The expansion-normalized dynamical variables suitable for the equations (9) - (12) are the following dimensionless combinations

$$
\begin{gathered}
u_{1}=\frac{\dot{R} f^{\prime \prime}}{f^{\prime} H}, u_{2}=\frac{R}{6 H^{2}}, u_{3}=\frac{f}{6 f^{\prime} H^{2}}, \\
u_{4}^{+}=\frac{\dot{\beta_{+}}}{4 H^{2}}, u_{4}^{-}=\frac{\dot{\beta_{-}}}{12 H^{2}}, u_{5}=\frac{\kappa \rho}{3 f^{\prime} H^{2}} .
\end{gathered}
$$

in terms of which the energy constraint $(9)$ reads simply

$$
g=1+u_{1}-u_{2}+u_{3}-u_{4}^{+}-u_{4}^{-}-u_{5}=0
$$

from where we have that one of the expansion-normalized variables can always be eliminated. Unless otherwise stated, we will always choose the matter content variable $u_{5}$ to be expressed in terms of the others dynamical variables. The variable

$$
u_{4}=u_{4}^{+}+u_{4}^{-}=\frac{\sigma^{2}}{6 H^{2}}
$$

is also relevant for our purposes. It is important to stress that the variables $u_{4}^{+}$and $u_{4}^{-}$are both non-negative by construction. Now, let us introduce the quantity

$$
\gamma(R)=\frac{f^{\prime}}{R f^{\prime \prime}},
$$

which, or course, contains the information about the form of $f(R)$. Knowing the form of $f(R), \gamma$ can be determined in terms of the dynamical variables $u_{2}, u_{3}$ by inverting the relation

$$
\frac{u_{2}}{u_{3}}=\frac{R f^{\prime}}{f} .
$$

We will return to the question of the invertibility of (21) in the last section. The 5-dimensional system of autonomous first order differential equations fully equivalent to $10-12$ is

$$
\begin{gathered}
\epsilon \frac{d u_{1}}{d N}=1+u_{2}-3 u_{3}-u_{4}-3 \omega u_{5} \\
-u_{1}\left(u_{1}+u_{2}-u_{4}\right) \\
\epsilon \frac{d u_{2}}{d N}=u_{1} u_{2} \gamma\left(\frac{u_{2}}{u_{3}}\right)-2 u_{2}\left(u_{2}-u_{4}-2\right) \\
\epsilon \frac{d u_{3}}{d N}=u_{1} u_{2} \gamma\left(\frac{u_{2}}{u_{3}}\right)-u_{3}\left(u_{1}+2 u_{2}-2 u_{4}-4\right) \\
\epsilon \frac{d u_{4}^{+}}{d N}=-2 u_{4}^{+}\left(1+u_{1}+u_{2}-u_{4}\right)+3 \mu_{+} \sqrt{u_{4}^{+}} u_{5} \\
\epsilon \frac{d u_{4}^{-}}{d N}=-2 u_{4}^{-}\left(1+u_{1}+u_{2}-u_{4}\right)+\mu_{-} \sqrt{3 u_{4}^{-}} u_{5} \\
\epsilon \frac{d u_{5}}{d N}=-u_{5}\left(3 \omega-1+u_{1}+2 u_{2}-2 u_{4}\right. \\
\left.+3 \mu_{+} \sqrt{u_{4}^{+}}+\mu_{-} \sqrt{3 u_{4}^{-}}\right)
\end{gathered}
$$

Notice that differentiating (18) with respect to $N$ and using the equations $22-28$, we have

$$
\epsilon \frac{d g}{d N}=-\left(u_{1}+2 u_{2}-2 u_{4}^{+}-2 u_{4}^{-}-1\right) g,
$$

showing that the constraint $g=0$ is indeed conserved along the solutions of our equations and the system 22 - 28) is effectively 5-dimensional.

The case of $f(R)=R^{1+\delta}$, with $\delta \neq 0$, will be particularly important in our next examples. For this choice of $f(R)$, one has simply

$$
\gamma=\delta^{-1}
$$

and the equations 23 and (24) can be considerably simplified. In this case, the right-handed side of the equations 22 - 28 involves only second degree polynomials in $u_{1}, u_{2}$, and $u_{3}$, and forth degree in $\sqrt{u_{4}^{-}}$ and $\sqrt{u_{4}^{+}}$. Hence, the task of finding the fixed points of our system reduce to finding polynomial roots, which may be performed in general with good accuracy. Notice 
that there are other relevant choices for $f(R)$ leading to polynomial fixed points. Besides of the trivial extension $f(R)=\alpha R^{1+\delta}+\Lambda$, with $\alpha$ and $\Lambda$ constants, for which 29 also holds. We have also the case $f(R)=\alpha \ln R+\Lambda$, which corresponds to $\delta \rightarrow-1$ in $(29)$. For the so-called exponential gravity 41 44, for which $f(R)=e^{\alpha R}$, we have

$$
\gamma=\frac{u_{3}}{u_{2}}
$$

and the polynomial nature of the fixed points if of course maintained. The same occurs to the well known case [14] $f(R)=R+\frac{\alpha}{R}$, for which

$$
\gamma=\frac{u_{2}}{u_{3}-u_{2}}
$$

This case belongs, in fact, to the more general class of functions $f(R)=R^{a}+\alpha R^{b}$, with $a \neq b$ constants, for which we have

$$
\gamma=\frac{u_{2}}{(b+a-1) u_{2}-a b u_{3}} .
$$

Notice that, as in the exponential case, the function $\gamma$ does not depend on the parameter $\alpha$. This, of course, does not mean that the dynamics in insensitive to the value of $\alpha$, since the expansion-normalized variables (17) depend explicitly on $\alpha$. The case $a=1$ and $b=2$ is the original Starobinsky inflationary scenario [7, and for the vacuum case our approach reduces to that one considered recently in [45. In the last section, we will discuss in more detail the vast class of functions $f(R)$ with polynomial fixed points.

\section{APPLICATIONS}

For the sake of illustration, we will consider some explicit examples for our approach. Some new results will be obtained, and some other well known results will be rederived in a simpler and more direct way. We will consider in this section the case of expanding universes $(\epsilon=1)$. Contracting universes $(\epsilon=-1)$ correspond to logarithmic time-reversed dynamics.

\section{A. $R^{1+\delta}$ vacuum solutions}

Our first example will be the case $f(R)=R^{1+\delta}$, whose main motivations from a cosmological perspective can be found in 30 33, for instance. The case with $\delta=0$ is obviously pure GR, for which the corresponding system is lower-dimensional, and our approach simply does not apply. The case logarithmic case $f(R)=\ln R$ must be treated separately. Hence, we will start considering $\delta \neq 0$ and $\delta \neq-1$. Since we will deal with vacuum solutions, we set $u_{5}=0$ in the equations $(18)$ and $(22)-(28)$. In this case, notice that 25 and 26 can be combined in only one equation for $u_{4}$. We can then use 18 to write $u_{3}$ as

$$
u_{3}=u_{2}-u_{1}+u_{4}-1
$$

and we are left with only three dynamical variables $u_{1}, u_{2}$, and $u_{4}$. Now, there is an interesting point to notice 22 about the specific choice $f(R)=R^{1+\delta}$, with $\delta \neq-1$, namely that

$$
\frac{u_{2}}{u_{3}}=\frac{R f^{\prime}}{f}=1+\delta,
$$

which combined with the constraint 33 implies

$$
\delta u_{2}=(1+\delta)\left(u_{1}-u_{4}+1\right),
$$

and we are left in fact with a two-dimensional phase space spanned by the variables $u_{1}$ and $u_{4}$. The corresponding dynamical equations in this case are

$$
\begin{aligned}
\frac{d u_{1}}{d N} & =\phi_{1}\left(u_{1}, u_{4}\right) \\
& =-\delta^{-1}(1+2 \delta)\left(u_{1}-u_{1}^{*}\right)\left(u_{1}-u_{4}+1\right) \\
\frac{d u_{4}}{d N} & =\phi_{4}\left(u_{1}, u_{4}\right) \\
& =-2 \delta^{-1}(1+2 \delta) u_{4}\left(u_{1}-u_{4}+1\right)
\end{aligned}
$$

where

$$
u_{1}^{*}=\frac{2(\delta-1)}{1+2 \delta} .
$$

The phase space $\left(u_{1}, u_{4}\right)$ associated with the system 36 - 37 has some interesting features. For instance, it has an one-dimensional invariant subspace (a continuous line of fixed points) corresponding to the straight line $u_{1}-$ $u_{4}=-1$. However, from 33 we have that $u_{3}=u_{2}$ on this line, which implies from (17) and (34) that $R=0$ on $u_{1}-u_{4}=-1$. Besides of this invariant straight line, we have also the isolated fixed $\left(u_{1}^{*}, 0\right)$, for $\delta \neq-\frac{1}{2}$. The case $\delta=-\frac{1}{2}$ will be also discussed separately.

The stability of the isolated fixed point can be inferred from the linearization of $(36)$ - (37). The Jacobian matrix of 36 - 37) at the point $\left(u_{1}^{*}, 0\right)$ reads

$$
\left(\frac{\partial\left(\phi_{1}, \phi_{4}\right)}{\partial\left(u_{1}, u_{4}\right)}\right)=-\delta^{-1}(4 \delta-1)\left(\begin{array}{ll}
1 & 0 \\
0 & 2
\end{array}\right),
$$

from where we have that such fixed point is stable for $\delta>\frac{1}{4}$ or for $\delta<0$. For the stability of the invariant straight line, we can consider the divergence of the vector field $\left(\phi_{1}, \phi_{2}\right)$. One has

$$
\nabla \cdot \boldsymbol{\phi}=\frac{\partial \phi_{1}}{\partial u_{1}}+\frac{\partial \phi_{4}}{\partial u_{4}}=\delta^{-1}\left((1+2 \delta) u_{4}+4 \delta-1\right)
$$

on the invariant line. Recalling that $u_{4} \geq 0$, we have that the invariant line is entirely repulsive (positive divergence) for $\delta>\frac{1}{4}$ or for $\delta \leq-\frac{1}{2}$. For $-\frac{1}{2}<\delta \leq \frac{1}{4}$, we can have some attractive segments, depending on the value of 


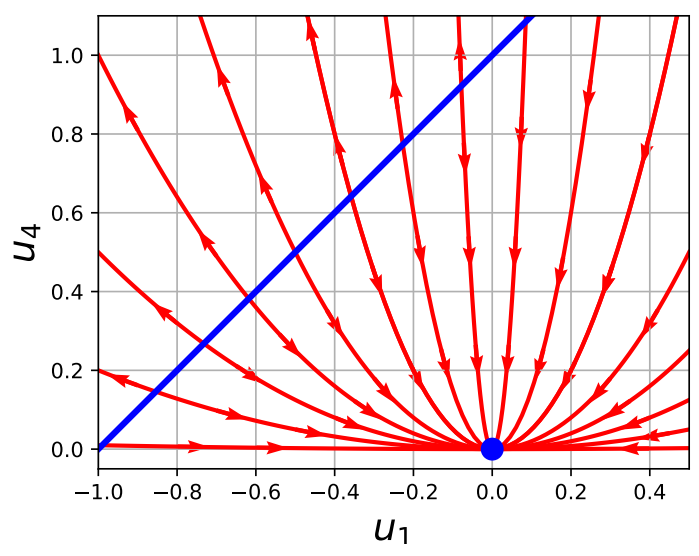

FIG. 1. Phase space for the system (36) - (37), for $\delta=1$. The fixed point $(0,0)$ is located in the semiplane below the critical line. The solutions are restricted to parabolas centered in the attractive fixed point. The region below the invariant line corresponds to the attraction basin of the fixed point. Any solution starting there will tend asymptotically to the fixed point. All solutions starting in the region above the critical line will diverge to infinity. Notice that the critical line is entirely repulsive. Such phase space is rather generic, it is essentially the same for all theories of the type $f(R)=R^{1+\delta}$ such that the fixed point is attractive and is located below the invariant line.

$u_{4}$. We will return to the physical interpretation of this $R=0$ invariant line in a following sub-section. The case $\delta=-\frac{1}{2}$ is particularly curious, since the isolated fixed point is absent and we have a second one-dimensional invariant line, namely $u_{1}=0$, which is also entirely repulsive. On the other hand, the case $f(R)=\ln R$ cannot be incorporated in the present analysis since (34) is not valid for $\delta \rightarrow-1$, and in fact we have a three-dimensional phase space for such case.

The solutions of (36) and (37) are curves on the plane $\left(u_{1}, u_{4}\right)$, and it turns out that such curves can be determined analytically. Notice that the solutions are such that

$$
\frac{u_{4}^{\prime}}{u_{1}^{\prime}}=\frac{2 u_{4}}{u_{1}-u_{1}^{*}}
$$

which can be integrated as

$$
u_{4}=c\left(u_{1}-u_{1}^{*}\right)^{2},
$$

with arbitrary $c$. Thus, the phase space trajectories of all solutions of (36) and (37) are simply parabolas centered in the isolated fixed point, irrespective of the value of $\delta$, provided the fixed point exists. Since we known the trajectories graphs, one can infer the dynamics direction and, consequently, the dynamical properties of the fixed point and the invariant line, directly form the equations (36) and (37) as follows. Consider the phase space function $L=u_{1}-u_{4}+1$. It is clear that $L=0$ is the invariant line. On the other hand, $L=c$ constant is a parallel line

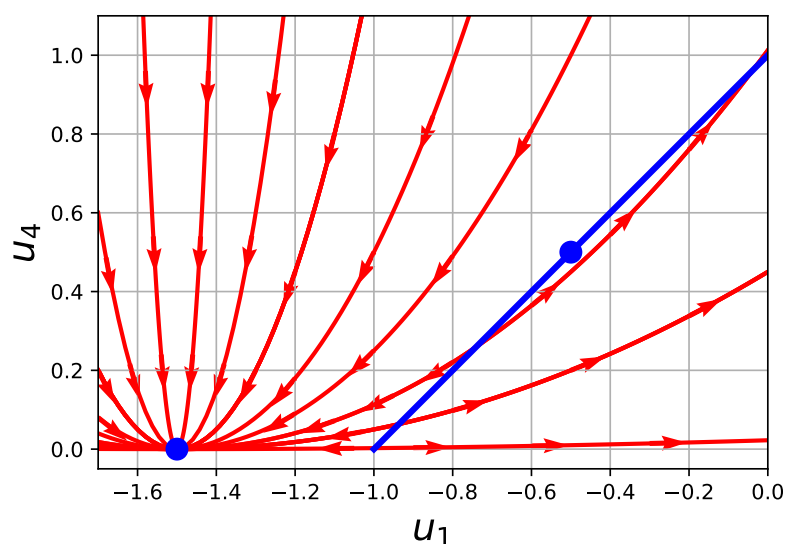

FIG. 2. Phase space for the system 36 - 37 , for $\delta=$ $\frac{1}{10}$. The fixed point $\left(-\frac{3}{2}, 0\right)$ is now located in the semiplane above the critical line. The solutions are also restricted to parabolas centered in the attractive fixed point. However, the attraction basin of the fixed point is now in the region above the critical line. Notice that the invariant line in this case has an attractive and a repulsive segment located, respectively, above and below the depicted point $\left(-\frac{1}{2}, \frac{1}{2}\right)$. The divergence (40) always vanishes in limit points between attractive and repulsive segments like this one.

located below the invariant line if $c>0$, or above if $c<0$. The invariant line is the boundary between two semiplanes with reverse dynamics direction, and the dynamical properties of the fixed point and of the invariant line depend on the relative position between then, see Figs 1 and 2, which correspond, respectively, to the cases $\delta=1$ and $\delta=\frac{1}{10}$. The former is the important case of the Starobinsky's inflationary scenario with $f(R)=R^{2}$, which we will discuss in more details in the next subsection.

Notice that knowing that the solutions are constrained to the parabolas (42), the exact solutions of (36) and (37) boils down to a simple quadrature of a rational function

$$
\frac{d \bar{u}_{1}}{c \bar{u}_{1}^{3}-\bar{u}_{1}^{2}-\left(u_{1}^{*}+1\right) \bar{u}_{1}}=-\delta^{-1}(1+2 \delta) d N
$$

with $u_{1}=\bar{u}_{1}+u_{1}^{*}$. For the case $\delta=-\frac{1}{2}, u_{4}$ is a constant and (36) also reduces to a simple rational quadrature. We have just established that the vacuum solutions for the $f(R)=R^{1+\delta}$ case, for $\delta \neq-1$, are exactly soluble.

Since the stable fixed points of a cosmological model correspond to the cosmological histories which will dominate the asymptotic evolution of the system, it worth to look more closely on them. By using (33) and (34), we have that the isolated fixed points are such that

$$
u_{3}=\frac{4 \delta-1}{\delta(1+2 \delta)},
$$

with $\delta>\frac{1}{4}$ or $\delta<0$. From the definition of $u_{3}$ and 5 , 
we have that 440 implies that

$$
\dot{H}=\Delta H^{2},
$$

where

$$
\Delta=\frac{\delta-1}{\delta(1+2 \delta)} .
$$

It is clear that for $\delta=1$, the stable fixed point corresponds to de Sitter solution with $a(t)=e^{H t}$, with constant $H$. (The case $H=0$ corresponds to the flat Minkowski spacetime). This is namely the well known Starobinsky's inflationary solution, which we will consider in more details in the next subsection. For $\delta \neq 1$, the solutions are

$$
H(t)=\frac{H_{0}}{1-\Delta H_{0}\left(t-t_{0}\right)},
$$

where $H\left(t_{0}\right)=H_{0}$, which interpretation is straightforward. For $\Delta>0$, which corresponds to $-\frac{1}{2}<\delta<0$ or $\delta>1$, we have a future finite time big rip singularity, while for $\Delta<0\left(\delta<-\frac{1}{2}\right.$ or $\left.0<\delta<1\right)$, the Hubble parameter $H$ decreases as $t^{-1}$ for large $t$, i.e., the solution asymptotically tends to a power law expansion.

\section{B. Uniqueness of Starobinsky's inflationary scenario}

From the last subsection, we have that the Starobinsky's $R^{2}$ inflationary scenario is unique among the $F(R)=R^{1+\delta}$ theories of gravity, since only for $\delta=1$ the stable de Sitter fixed point $(0,0)$ is available, a result indeed known for a long time, see [34 36], for instance. We can, however, easily prove a stronger result for generic $f(R)$ theories. The de Sitter solution $a(t)=e^{H t}$, with constant and arbitrary $H$, implies $u_{1}=u_{4}=0$, and also

$$
R=12 H^{2},
$$

which, on the other hand, determine that $u_{2}=2$ and $u_{3}=\frac{2 f}{R f^{\prime}}$ and, hence, the constraint 18 will read

$$
R f^{\prime}(R)=2 f(R) .
$$

Since we assume that de Sitter solution exists for arbitrary $H$, we have from 48 that it should exist for any $R>0$, and hence equation $(49)$ can be seen as a ordinary differential equation for $f(R)$, which unique solution is $f(R)=\alpha R^{2}$, establishing in this way a stronger result: the case $R^{2}$ is unique among all vacuum $f(R)$ theories with respect to the existence of a de Sitter solution with arbitrary $H$. The condition 49) was first obtained by Barrow and Ottewill in 34 by using a more intricate approach, but here we see that it appears from a very simple analysis of fixed points. We will return to this problem in the last section.

\section{Kasner-type solutions}

The third example that we wish to consider is the vacuum Kasner-type solution of the form

$$
d s^{2}=-d t^{2}+\left(t^{p_{1}} d x^{1}\right)^{2}+\left(t^{p_{2}} d x^{2}\right)^{2}+\left(t^{p_{3}} d x^{3}\right)^{2} .
$$

The Ricci scalar, the average Hubble constant, and the total anisotropy $\sigma$ for such metric read, respectively,

$$
\begin{aligned}
R & =\frac{2}{t^{2}}(Q+S-P), \quad H=\frac{P}{3 t}, \quad \text { and } \\
\sigma^{2} & =\frac{1}{3 t^{2}}\left(3 Q-P^{2}\right),
\end{aligned}
$$

where

$$
\begin{aligned}
& Q=p_{1}^{2}+p_{2}^{2}+p_{3}^{2}, \\
& S=p_{1} p_{2}+p_{1} p_{3}+p_{2} p_{3}, \\
& P=p_{1}+p_{2}+p_{3} .
\end{aligned}
$$

We will consider here the case $f(R)=R^{1+\delta}$, exactly in the same line of [32, 33]. In terms of our expansionnormalized variables, the Kasner solutions in this case corresponds to following fixed points

$$
\begin{aligned}
& u_{1}=-\frac{6 \delta}{P}, \\
& u_{2}=\frac{3(Q+S-P)}{P^{2}}, \\
& u_{3}=\frac{3(Q+S-P)}{(1+\delta) P^{2}}, \\
& u_{4}=\frac{3 Q-P^{2}}{2 P^{2}}
\end{aligned}
$$

Notice that we already know from the first example the complete phase space for the vacuum $f(R)=R^{1+\delta}$ theory. It has a fixed isolated point and an invariant straight line. The fixed point corresponds to an isotropic solution $\left(u_{4}=0\right)$ and imposing the condition $u_{1}=u_{1}^{*}$, we have

$$
p_{1}=p_{2}=p_{3}=\frac{\delta(1+2 \delta)}{1-\delta}
$$

which is a well known FLRW type solution for $R^{1+\delta}$ gravity [46 48]; see also [32] for further discussions. All possible anisotropic Kasner solutions must be necessarily on the zero curvature invariant line where $u_{2}=u_{3}=0$, implying that

$$
Q+S-P=0 .
$$

The invariant line $u_{1}-u_{4}+1=0$ in this case reads

$$
12 \delta P+3 Q-3 P^{2}=0 .
$$

Notice that there is another algebraic relation valid for all $P, Q$, and $S$, namely

$$
P^{2}-Q-2 S=0 .
$$


Solving the equations 60, 61, and 62 for $P, Q$, and $S$ gives

$$
\begin{aligned}
& P=2 \delta+1 \\
& Q=(2 \delta+1)(1-2 \delta), \\
& S=2 \delta(2 \delta+1)
\end{aligned}
$$

Since $Q \geq 0$, we have from 64 that the existence of a Kasner solution requires $-\frac{1}{2} \leq \delta \leq \frac{1}{2}$, where both limits correspond to the Minkowski spacetime $\left(p_{1}=p_{2}=p_{3}=\right.$ $0)$. However, there is another more restrictive condition, namely the positiveness of $u_{4}$ given by 58

$$
3 Q-P^{2}=2(2 \delta+1)(1-4 \delta) \geq 0,
$$

from where we have $-\frac{1}{2} \leq \delta \leq \frac{1}{4}$, which is exactly Barrow and Clifton's result, originally obtained in a more intricate way in $[32,33$. The stability of these solutions is a quite interesting issue. First, notice that $\nabla \cdot \phi=0$ at the point $\left(-\frac{6 \delta}{1+2 \delta}, \frac{1-4 \delta}{1+2 \delta}\right)$ of the invariant line, which means that we need to go further the linear analysis in this case. However, the restriction for the existence of this Kassner-like solution implies that we are in a situations as depicted in Fig. 2, with the fixed point above the invariant line. It turns out that the Kassner-like solution always corresponds to the limit point of the attractive and repulsive segments of the invariant line(!), implying that both eigenvalues of the Jacobian matrix at this point vanish. However, it is clear the non-linear instability of this point, since any point above the invariant line is in the attraction basin of the fixed point.

\section{Fixed points with anisotropic matter}

As an example of application of the full set of our expansion-normalized variables, let us consider the case of $f(R)$ theories with polynomials fixed points. The simplest case is our example of a $f(R)=R^{1+\delta}$ theory, but now with an anisotropic barotropic fluid of the type (7). Taking into account 29 and $(34)$, the full set of equations in this case will be

$$
\begin{gathered}
\frac{d u_{1}}{d N}=1+(\delta-2) u_{3}-u_{4}-3 \omega u_{5} \\
-u_{1}\left(u_{1}+(1+\delta) u_{3}-u_{4}\right), \\
\frac{d u_{3}}{d N}=u_{3}\left(\delta^{-1} u_{1}-2(1+\delta) u_{3}+2 u_{4}+4\right), \\
\frac{d u_{4}^{+}}{d N}=-2 u_{4}^{+}\left(1+u_{1}+(1+\delta) u_{3}-u_{4}\right) \\
+3 \mu_{+} \sqrt{u_{4}^{+}} u_{5}, \\
\frac{d u_{4}^{-}}{d N}=-2 u_{4}^{-}\left(1+u_{1}+(1+\delta) u_{3}-u_{4}\right) \\
+\mu_{-} \sqrt{3 u_{4}^{-}} u_{5},
\end{gathered}
$$

recalling that $u_{4}=u_{4}^{+}+u_{4}^{-}$, and

$$
u_{5}=1+u_{1}-\delta u_{3}-u_{4} \text {. }
$$

We are particularly interested in the isotropic fixed points, i.e., the solutions with $u_{4}^{+}=u_{4}^{-}=0$. It turns out that there exist four isolated fixed points of this type, namely the following values for the pair $\left(u_{1}, u_{3}\right)$

$$
\begin{aligned}
(-1,0), & (1-3 \omega, 0), \quad\left(\frac{2(\delta-1)}{1+2 \delta}, \frac{4 \delta-1}{\delta(1+2 \delta)}\right), \\
& \left(-\frac{3 \delta(\omega+1)}{1+\delta}, \frac{4 \delta+1-3 \omega}{2(1+\delta)^{2}}\right) .
\end{aligned}
$$

The relevant question here is whether some of these fixed points are attractive, which would correspond to asymptotically stable isotropic solutions in the presence of anisotropic matter. We will show that for anisotropic fluids, all isotropic fixed points are unstable. Nevertheless, for isotropic fluids $\left(\mu_{+}=\mu_{-}=0\right)$, in principle, some of the isotropic fixed points could be indeed stable.

In order order to prove that the system 67 - 70 do not admit any stable isotropic fixed point in the presence of anisotropic matter, let us assume, without loss of generality, that $\mu_{+}>0$, and consider 69 near a generic isotropic fixed point $\left(u_{1}, u_{3}, u_{4}^{+}, u_{4}^{-}\right)=\left(u_{1}^{*}, u_{3}^{*}, 0,0\right)$,

$$
\frac{d u_{4}^{+}}{d N}=\sqrt{u_{4}^{+}}\left(3 \mu_{+} u_{5}^{*}-2 \sqrt{u_{4}^{+}}\left(1+u_{1}^{*}+(1+\delta) u_{3}^{*}\right)\right) \text {, }
$$

where $u_{5}^{*}=1+u_{1}^{*}-\delta u_{3}^{*}$ is the matter content associated with the fixed point. For the case of a barotropic anisotropic fluid (7), it is natural to assume $u_{5}^{*}>0$. Since $\mu_{+} u_{5}^{*}>0$, it is clear that there is a neighborhood of $u_{4}^{+}=0$ where the right-handed side of 73 is positive, implying the repulsiveness of the isotropic fixed points at least along the positive $u_{4}^{+}$direction. For the case of an isotropic fluid, since $\mu_{+}=\mu_{-}=0$, one can have attractive fixed points according to the sign of the term between parenthesis. Nevertheless, for anisotropic fluids, no isotropic fixed point can be stable. It is important to stress that, from the structure of the equations 22 (28), we see that the same conclusion will hold for any choice of $f(R)$ with polynomial fixed points, since we will always have a repulsive neighborhood of $u_{4}^{+}=0$ as we had in 73.

\section{E. Exponential gravity}

As a last example for our dynamical approach, let us consider the case of exponential gravity $f(R)=e^{\alpha R}$, $\alpha \neq 0$, which main motivations and implications in cosmology can be found, for instance, in [4] 44]. Taking into account (30), we will have in this case the 5-dimensional system given by the equations $22-26$, but now with the following governing equations for $u_{2}$ and $u_{3}$

$$
\begin{aligned}
& \frac{d u_{2}}{d N}=u_{1} u_{3}-2 u_{2}\left(u_{2}-u_{4}-2\right), \\
& \frac{d u_{3}}{d N}=-2 u_{3}\left(u_{2}-u_{4}-2\right),
\end{aligned}
$$


recalling that $u_{4}=u_{4}^{+}+u_{4}^{-}$. Notice that for the exponential gravity, the phase space variable $u_{3}$ reads

$$
u_{3}=\frac{1}{6 \alpha H^{2}}
$$

and, hence, it ranges over $(0, \infty)$ and $(-\infty, 0)$, respectively, for $\alpha>0$ and $\alpha<0$. The variable $u_{4}$ is nonnegative and all other variables can assume any real value. This is the phase space for the exponential gravity theory.

Let us consider first the vacuum case, for which $u_{5}=0$, implying that equations 25 and (26) can be combined into a single equation for $u_{4}$, and that $u_{2}$ can be eliminated by using (18), leading finally to the following 3dimensional system

$$
\begin{aligned}
& \frac{d u_{1}}{d N}=2+u_{1}-2 u_{3}-2 u_{4} \\
& \quad-u_{1}\left(1+2 u_{1}+u_{3}-2 u_{4}\right), \\
& \frac{d u_{3}}{d N}=2 u_{3}\left(1-u_{1}-u_{3}+2 u_{4}\right), \\
& \frac{d u_{4}}{d N}=-2 u_{4}\left(2+2 u_{1}+u_{3}-2 u_{4}\right) .
\end{aligned}
$$

It is clear from (77) - 79p that the boundary plane $u_{3}=0$ is an invariant subspace, implying that no solution will ever reach it in finite time. Any solution on this invariant subspace can be only reached asymptotically for $N \rightarrow \infty$. A fixed-point analysis of our system reveals the existence of the following fixed points $\left(u_{1}, u_{3}, u_{4}\right)$ :

$$
\left(u_{4}-1,0, u 4\right), \quad(1,0,0), \quad(0,1,0) .
$$

The first solution is exactly the same invariant subspace we have already discussed in our first two examples, which in the present case is entirely contained in the invariant boundary $u_{3}=0$. The other two solutions are isolated isotropic fixed points whose cosmological history can be reconstructed from (5) and, for instance, from the definition of $u_{2}$ given by (17), which implies

$$
\dot{H}=\left(u_{2}-2\right) H^{2} .
$$

Taking into account (18), we have that that the invariant line corresponds to a cosmological history like (47), while both isolated fixed points are de Sitter solutions. The respective eigenvalues for these fixed points are

$$
(-8,-4,0) \text { and }\left(-6,-\frac{3+\sqrt{17}}{2}, \frac{\sqrt{17}-3}{2}\right) \text {, }
$$

revealing that the fixed point $(0,1,0)$ is clearly unstable (a saddle point). For the $(1,0,0)$ fixed point at the boundary, the indifferent direction is $\boldsymbol{v}=(3,-4,0)$. However, from a closer inspection of the system 77 78 on the line $(1+3 s,-4 s, 0)$

$$
\boldsymbol{v} \cdot \frac{d \boldsymbol{u}}{d N}=3 \frac{d u_{1}}{d N}-4 \frac{d u_{3}}{d N}=14 s^{2},
$$

we conclude that such fixed point $(s=0)$ will be indeed stable for $u_{3}>0(\alpha>0)$ and unstable for $u_{3}<0(\alpha<0)$. These results are entirely compatible with the spatially flat case of those ones obtained in [41] for the isotropic case of exponential gravity.

Let us now consider the case of exponential gravity in the presence of anistropic matter of the type (7), in the same line we have followed in the preceding subsection. We are also interested here in the isotropic fixed points $u_{4}^{+}=u_{4}^{-}=0$. The dynamical equations have five isolated isotropic fixed points in this case, namely the values of $\left(u_{1}, u_{2}, u_{3}\right)$ given by

$$
\begin{gathered}
(1,2,0), \quad(-3(1+\omega), 2,0), \\
(-1,0,0), \quad(1-3 \omega, 0,0), \quad(0,2,1) .
\end{gathered}
$$

As in the previous example, the relevant question here is whether any of these fixed points is attractive, which would correspond to an asymptotically stable isotropic solution in the presence of anisotropic matter. It turns out that exactly the same results of the preceding subsection hold here, since the structure of the equations for $u_{4}^{+}$and $u_{4}^{-}$are essentially the same for exponential gravity and for any theory with polynomial fixed points as, for instance, $f(R)=R^{1+\delta}$. All isotropic fixed points for exponential gravity in the presence of an anisotropic barotropic fluid are unstable, even though for isotropic fluids $\left(\mu_{+}=\mu_{-}=0\right)$ one, in principle, might have some stable isotropic fixed points.

\section{FINAL REMARKS}

We have introduced a new set of expansion-normalized variables for homogeneous and anisotropic Bianchi-I spacetimes in $f(R)$ gravity in the presence of anisotropic matter. In terms of these new dynamical variables, the full set of Einstein's equations boils down to a 5dimensional phase space. As applications of the proposed dynamical approach, we have considered explicitly the $f(R)=R^{1+\delta}$ modified theory of gravity, and shown that its vacuum dynamics is exactly solvable. We have reobtained, in a easier and more direct way, several well known results for this particular choice of $f(R)$, as, for instance, Bleyer and Schmidt isotropic solutions 46 48. and Barrow and Clifton anisotropic ones 32, 33. We have also extended a uniqueness result for Starobisnki inflationary scenario, namely that the case $R^{2}$ is unique among all vacuum $f(R)$ theories with respect to the existence of a de Sitter solution with arbitrary $H$, a result obtained previously by Barrow and Ottewill by using a more intricate approach 34. Finally, we explore our full set of equations and demonstrate that, in the presence of anisotropic barotropic fluids of the type (7), no isotropic fixed point can be stable for $f(R)$ theories gravity with polynomials fixed points. The case of exponential gravity [41 44] was also explicitly treated.

There are several possibilities of applications for our dynamical formulation. For instance, we could extend 
the results on the existence of de Sitter solution of Section III B for other situations. Goedel and Einstein universes are natural candidates, since there already exist some existence results in the literature 49. We could also consider different geometric situations as, for instance, the case of Bianchi-IX metrics [50] or the presence of torsion [51, 52. Some of these points are now under investigation.

As a final remark, let us consider the two related issues left behind in the previous analysis, namely the full set of function $f(R)$ leading to polynomial fixed points, and the question of the invertibility of (34). Firstly, notice that the condition

$$
\gamma=\frac{f^{\prime}}{R f^{\prime \prime}}=c\left(\frac{u_{2}}{u_{3}}\right)^{q}=c\left(\frac{R f^{\prime}}{f}\right)^{q}
$$

with rational $q$ and $c$ constants, is sufficient to assure that the fixed points of the system 22 - 28 will be polynomial roots. The cases we have considered, $f(R)=R^{1+\delta}$ and $f(R)=e^{\alpha R}$, correspond, respectively, to the choices $q=0, c=\delta^{-1}$ and $q=-1, c=1$. However, equation 85 can be solved for any $q$ and $c$, giving origin to a large class $f(R)$ theories with polynomials roots. The exact solution also boils down to a quadrature of fractional functions, as one can see by substituting $R=e^{\rho}$ and $f=e^{\int h d \rho}$ in 85 , leading to the separable equation

$$
h^{\prime}=c^{-1} h^{1-n}-h^{2}+h,
$$

where the tilde denotes differentiation with respect to $\rho$. Notice that for all $f(R)$ theories such that $(85)$, we have no problem with the invertibility of (34). However, this is a real issue if the expression (34), as a function of $R$, fails to be monotonic. In this case, we would have distinct function $\gamma\left(u_{2}, u_{3}\right)$ and, consequently, different equations of motion according to the value of $R$, which would complicate considerably the dynamics of the system.

\section{ACKNOWLEDGMENTS}

The authors thank S. Pal and J.D. Barrow for enlightening discussions, and the Yukawa Institute for Theoretical Physics at Kyoto University, Kyoto, Japan, for the warm hospitality during the long-term workshop YITP-T-17-02 "Gravity and Cosmology 2018", where this work was initiated. A.S. is also grateful to FAPESP (grant 2013/09357-9) and CNPq for the financial support. The work of K.B. was partially supported by the JSPS KAKENHI Grant Number JP 25800136 and Competitive Research Funds for Fukushima University Faculty (18RI009).
[1] V. F. Mukhanov, H. A. Feldman and R. H. Brandenberger, Phys. Rept. 215, 203 (1992).

[2] A. Riotto, ICTP Lect. Notes Ser. 14, 317 (2003) hep$\mathrm{ph} / 0210162$.

[3] J. Wainwright and G.F.R. Ellis, Dynamical systems in cosmology, Cambridge University Press (1997).

[4] S. Bahamonde, C.G. Boehmer, S. Carloni, E.J. Copeland, W. Fang, and N. Tamanini, Phys. Rep. 775, 1 (2018). arXiv:1712.03107.

[5] A. H. Guth, Phys. Rev. D 23, 347 (1981).

[6] A. D. Linde, Phys. Lett. 108B, 389 (1982).

[7] A. A. Starobinsky, Phys. Lett. 91B, 99 (1980).

[8] N. Aghanim et al. [Planck Collaboration], Planck 2018 results. VI. Cosmological parameters. arXiv:1807.06209.

[9] Y. Akrami et al. [Planck Collaboration], Planck 2018 results. X. Constraints on inflation. arXiv:1807.06211.

[10] K. Bamba and S. D. Odintsov, Symmetry 7, 220 (2015). arXiv:1503.00442

[11] K. Bamba, S. Capozziello, S. Nojiri and S.D. Odintsov, Astrophys. Space Sci. 342, 155 (2012). arXiv:1205.3421

[12] S. Capozziello, S. Carloni and A. Troisi, Recent Res. Dev. Astron. Astrophys. 1, 625 (2003). astro-ph/0303041

[13] S. Nojiri and S. D. Odintsov, Phys. Rev. D 68, 123512 (2003). hep-th/0307288

[14] S. M. Carroll, V. Duvvuri, M. Trodden and M. S. Turner, Phys. Rev. D 70, 043528 (2004). astro-ph/0306438

[15] A. de Felice and S. Tsujikawa, Living Rev. Rel. 13 , 3 (2010). arXiv:1002.4928

[16] T.P. Sotiriou and V. Faraoni, Rev. Mod. Phys. 82, 451 (2010). arXiv:0805.1726
[17] V. Faraoni and S. Capozziello, Fundam. Theor. Phys. 170 (2010).

[18] S. Nojiri and S. Odintsov, Phys. Rep. 505, 59 (2011). arXiv:1011.0544

[19] S. Capozziello and M. De Laurentis, Phys. Rept. 509, 167 (2011). arXiv:1108.6266

[20] Y. F. Cai, S. Capozziello, M. De Laurentis and E. N. Saridakis, Rept. Prog. Phys. 79, no. 10, 106901 (2016). arXiv:1511.07586

[21] S. Nojiri, S. Odintsov, and V.K. Oikonomou, Phys. Rep. 692, 1 (2017). arXiv:1705.11098

[22] S. Carloni, P. K. S. Dunsby, S. Capozziello and A. Troisi, Class. Quant. Grav. 22, 4839 (2005). gr-qc/0410046

[23] L. Amendola, R. Gannouji, D. Polarski and S. Tsujikawa, Phys. Rev. D 75, 083504 (2007). gr-qc/0612180.

[24] S. Carloni, A. Troisi and P. K. S. Dunsby, Gen. Rel. Grav. 41, 1757 (2009). arXiv:0706.0452

[25] S. Carloni, JCAP 1509, 013 (2015). arXiv:1505.06015.

[26] A. Alho, S. Carloni, and C. Uggla, JCAP 08, 064 (2016). arXiv:1607.05715

[27] S.D. Odintsov and V.K. Oikonomou, Phys. Rev. D 96, 104049 (2017). arXiv:1711.02230

[28] S.D. Odintsov and V.K. Oikonomou, Phys. Rev. D 98, 024013 (2018). arXiv:1806.07295

[29] S.S. da Costa, F.V. Roig, J.S. Alcaniz, S. Capozziello, M. de Laurentis, M. Benetti, Class. Quantum Grav. 35, 075013 (2018). arXiv:1802.02572

[30] J. A. Leach, S. Carloni and P. K. S. Dunsby, Class. Quant. Grav. 23, 4915 (2006). gr-qc/0603012 
[31] N. Goheer, J. A. Leach and P. K. S. Dunsby, Class. Quant. Grav. 24, 5689 (2007). arXiv:0710.0814

[32] J. D. Barrow and T. Clifton, Class. Quant. Grav. 23, L1 (2006). gr-qc/0509085

[33] T. Clifton and J. D. Barrow, Class. Quant. Grav. 23, 2951 (2006). gr-qc/0601118

[34] J.D. Barrow. and A.C. Ottewill, J. Phys. A16, 2757 (1983).

[35] K. Maeda, Phys. Rev. D 37, 858 (1988).

[36] J. D. Barrow and S. Hervik, Phys. Rev. D 74, 124017 (2006). gr-qc/0610013

[37] T. S. Pereira, C. Pitrou and J. P. Uzan, JCAP 0709, 006 (2007). arXiv:0707.0736

[38] S. Chakraborty, Phys. Rev. D 98, 024009 (2018). arXiv:1803.01594

[39] S. Chakraborty, S. Pal, and A. Saa, Phys. Rev. D 99, 024020 (2019). arXiv:1812.01694

[40] O. Akarsu and C.B. Kilinc, Astr. Sp. Science, 326, 315 (2010). arXiv:1001.0550

[41] M. Abdelwahab, S. Carloni, and P.K.S. Dunsby, Class. Quantum Grav. 25, 135002 (2008). arXiv:0706.1375.

[42] E.V. Linder, Phys. Rev. D 80, 123528 (2009). arXiv:0905.2962
[43] S. I. Kruglov, Int. J. Mod. Phys. A 28, 13501194 (2013). arXiv:1204.6709

[44] S.D. Odintsov, D. Saez-Chillon Gomez, G.S. Sharov, Eur. Phys. J. C 77, 862 (2017). arXiv:1709.06800

[45] D. Muller, A. Ricciardone, A.A. Starobinsky, A. Toporensky, Eur. Phys. J. C 78, 311 (2018). arXiv:1710.08753

[46] U. Bleyer and H.J. Schmidt, Int. J. Mod. Phys. A5, 4671 (1990).

[47] H.J. Schmidt, Astron. Nachr. 311, 165 (1990). arXiv:grqc/0109004

[48] H.J. Schmidt, Lectures on Mathematical Cosmology. arXiv:gr-qc/0407095.

[49] T. Clifton and J.D. Barrow, Phys. Rev. D72, 123003 (2005). gr-qc/0511076

[50] J. D. Barrow and H. Sirousse-Zia, Phys. Rev. D39, 2187 (1989).

[51] L. Jarv, A.V. Toporensky, Phys. Rev. D 93, 024051 (2016). arXiv:1511.03933

[52] M.A. Skugoreva, A.V. Toporensky, Eur. Phys. J. C 78, 377 (2018). arXiv:1711.07069 